\title{
Morphology study of methane hydrate formation and dissociation in the presence of amino acid
}

\author{
Hari Prakash Veluswamy ${ }^{l}$, Qi Wei Hong ${ }^{1}$ and Praveen Linga ${ }^{1} *$ \\ ${ }^{1}$ Department of Chemical and Biomolecular Engineering, National University of Singapore, \\ Singapore 117585
}

Number of pages: 6

Number of Figures: 5

*corresponding author: e-mail: praveen.linga@nus.edu.sg; 


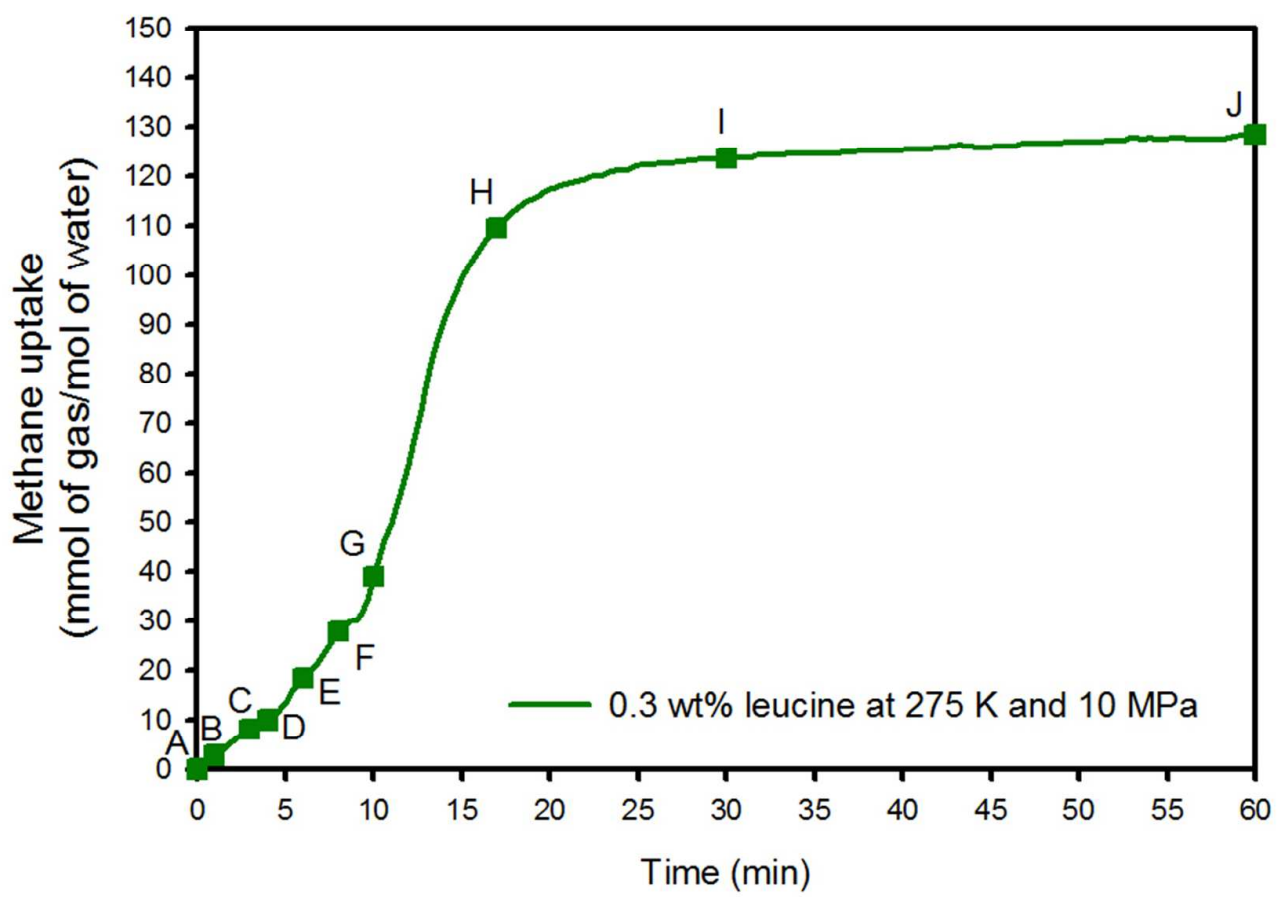

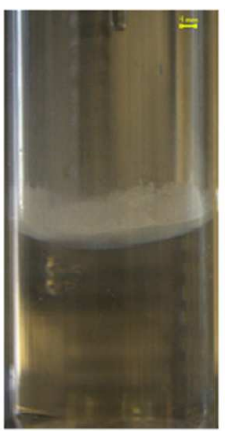

(A) at IT

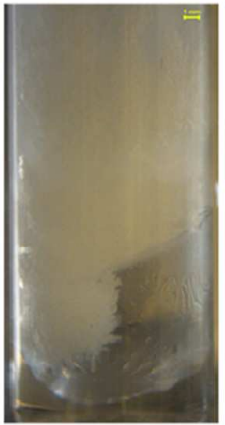

(F) 8 min from IT

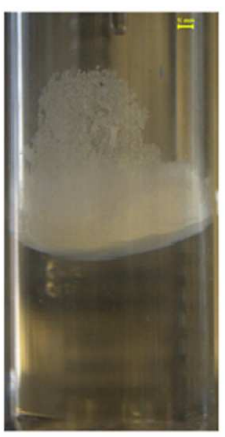

(B) 1 min from IT

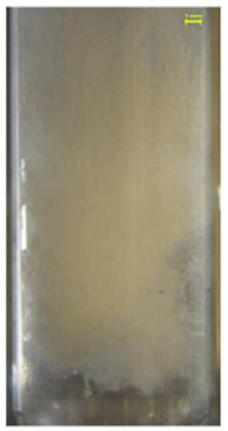

(G) 10 min from IT

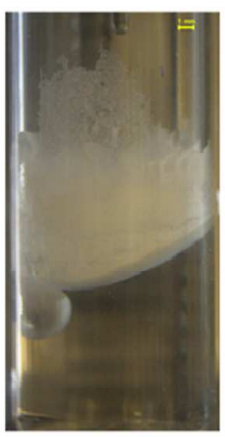

(C) 3 min from IT

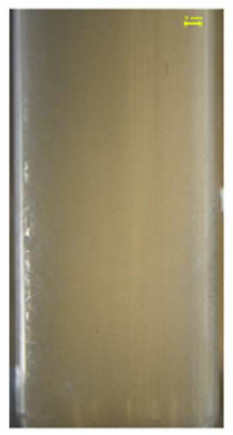

(H) 17 min from IT

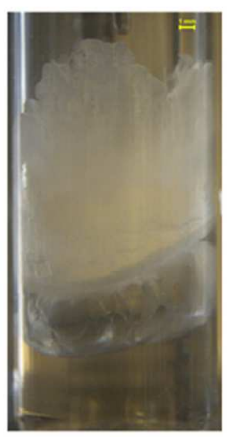

(D) 4 min from IT

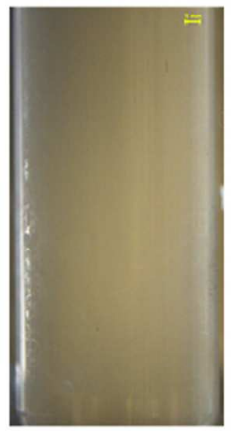

(I) $30 \mathrm{~min}$ from IT

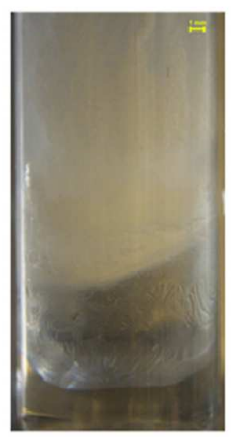

(E) 6 min from IT

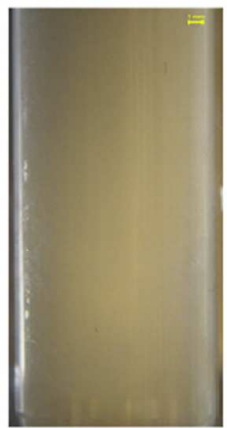

(J) 60 min from IT

Figure S1. Methane uptake data along with the visual observations during methane hydrate formation in presence of $0.3 \mathrm{wt} \%$ leucine 


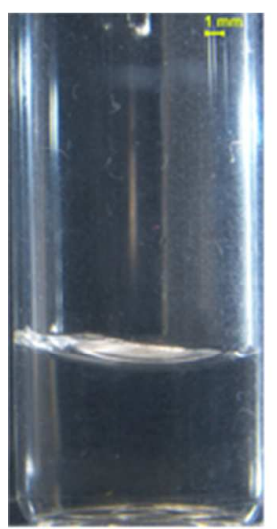

(a) Start of the experiment

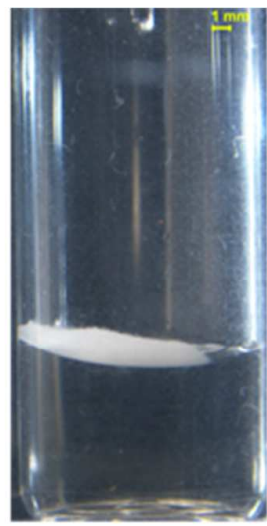

(b) $\mathrm{t}=0 \mathrm{~min}$ (induction)

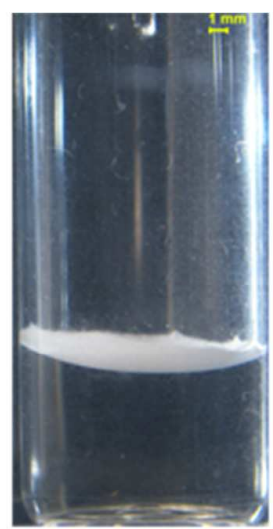

(c) $\mathrm{t}=2 \mathrm{~min}$

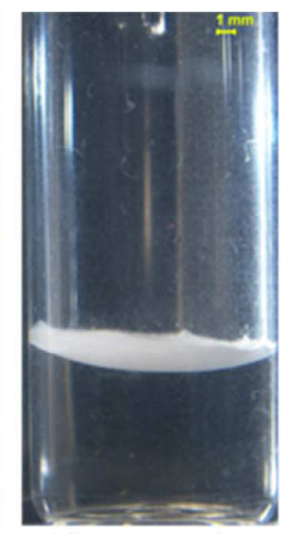

(d) $\mathrm{t}=5 \mathrm{~min}$

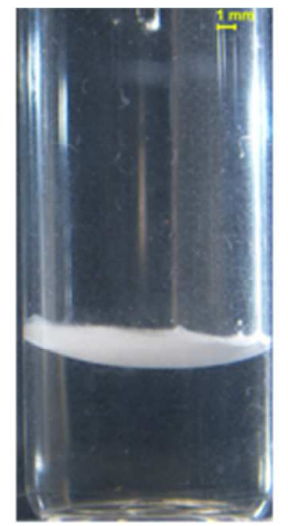

(e) $\mathrm{t}=10 \mathrm{~min}$

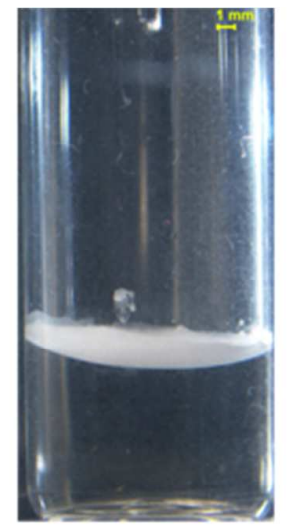

(f) $\mathrm{t}=30 \mathrm{~min}$

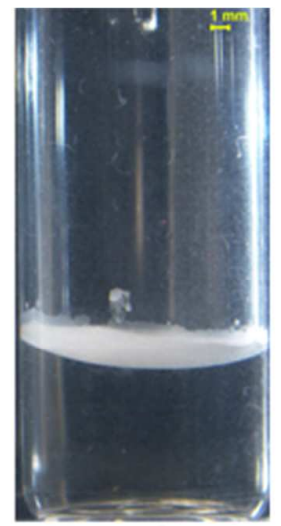

(g) $\mathrm{t}=60 \mathrm{~min}$

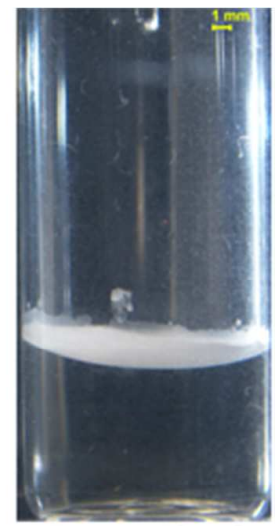

(h) $\mathrm{t}=240 \mathrm{~min}$

Figure S2. Morphology observations during the repeat trial of methane hydrate formation using $0.2 \mathrm{wt} \%$ leucine solution 


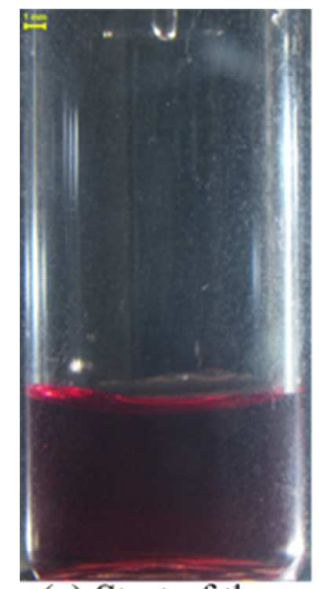

(a) Start of the experiment

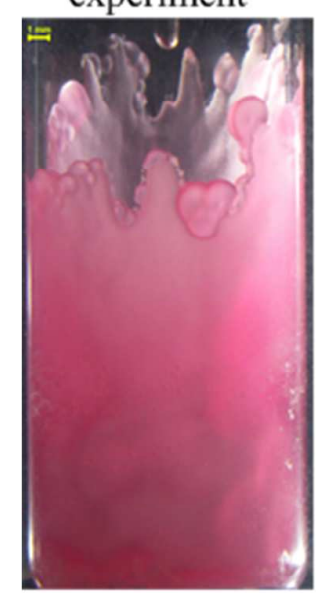

(h) $\mathrm{t}=3 \mathrm{~min}$

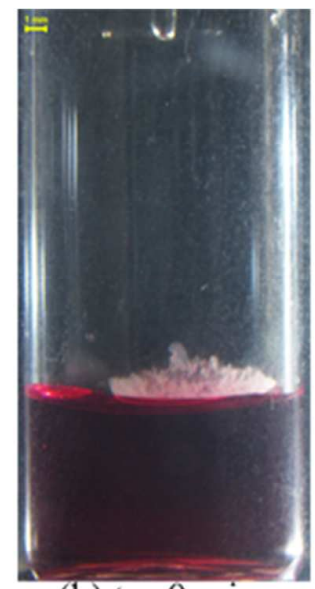

(b) $\mathrm{t}=0 \mathrm{~min}$ (induction)

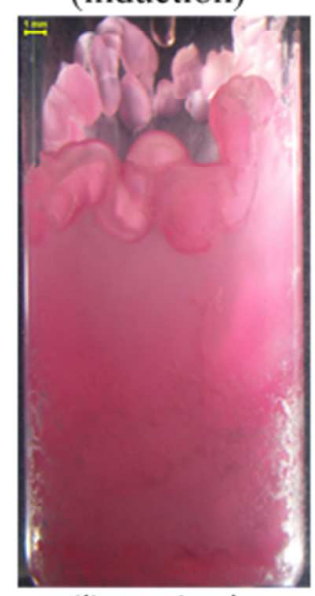

(i) $\mathrm{t}=4 \mathrm{~min}$

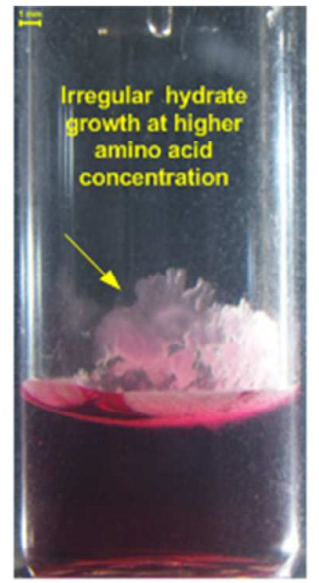

(c) $\mathrm{t}=0.3 \mathrm{~min}$

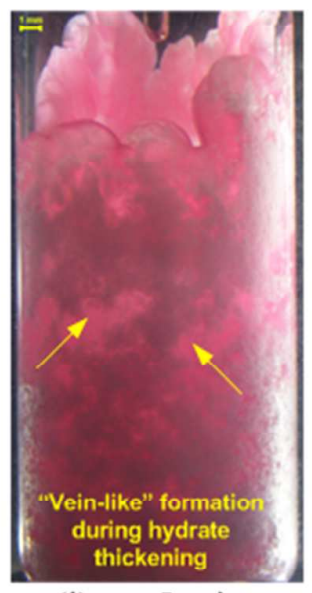

(j) $\mathrm{t}=5 \mathrm{~min}$

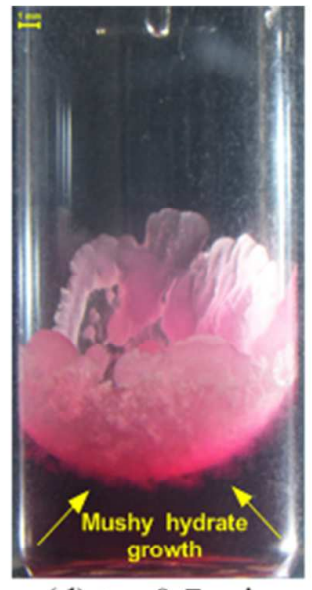

(d) $\mathrm{t}=0.7 \mathrm{~min}$

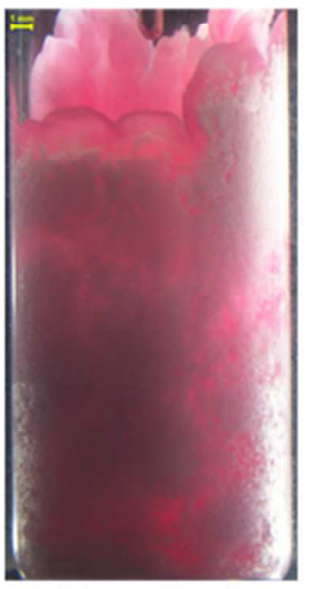

(k) $\mathrm{t}=8 \mathrm{~min}$

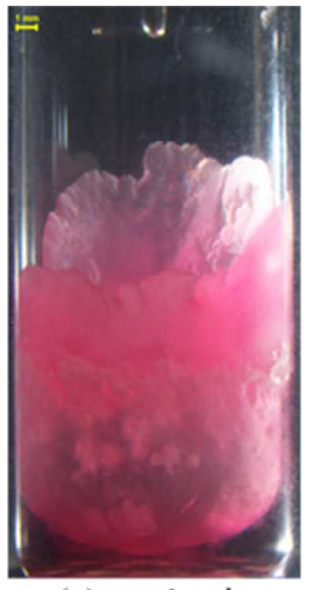

(e) $\mathrm{t}=1 \mathrm{~min}$

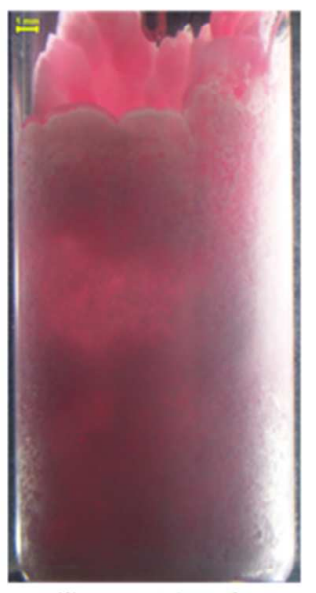

(1) $\mathrm{t}=10 \mathrm{~min}$

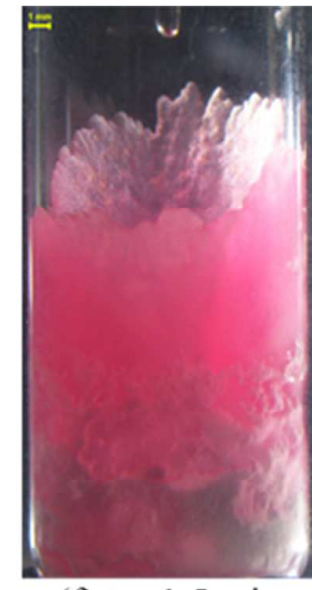

(f) $\mathrm{t}=1.5 \mathrm{~min}$

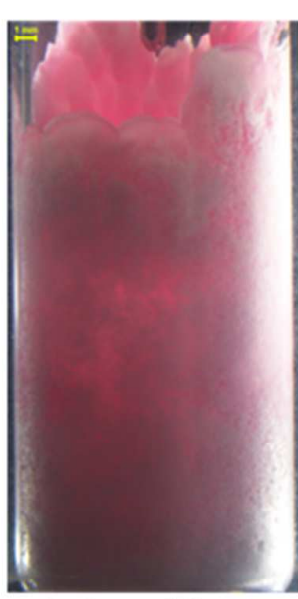

(m) $\mathrm{t}=30 \mathrm{~min}$

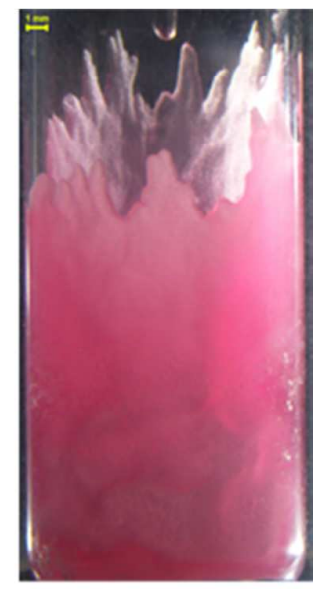

(g) $\mathrm{t}=2 \mathrm{~min}$

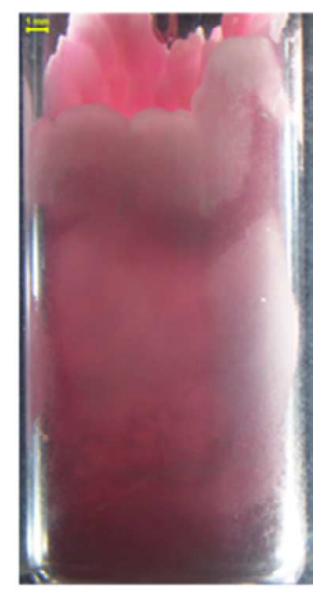

(n) $\mathrm{t}=180 \mathrm{~min}$

Figure S3. Morphology observations during methane hydrate formation using $0.5 \mathrm{wt} \%$ leucine solution in presence of red dye. 


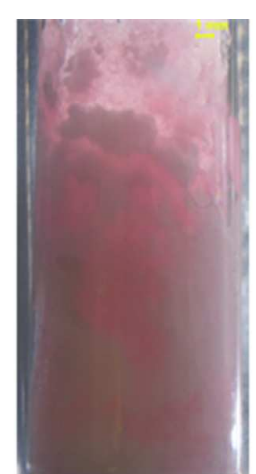

(a) Start of the decomposition

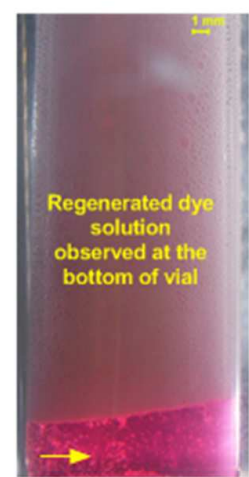

(g) t=120 min

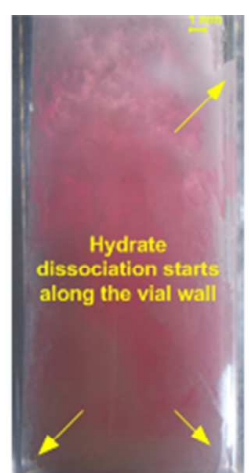

(b) t=15 min

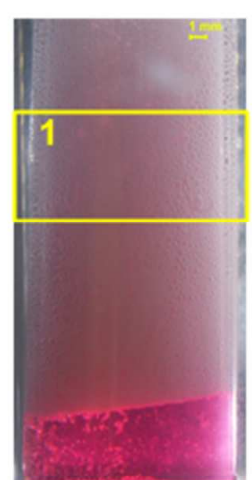

(h) t=180 $\min$

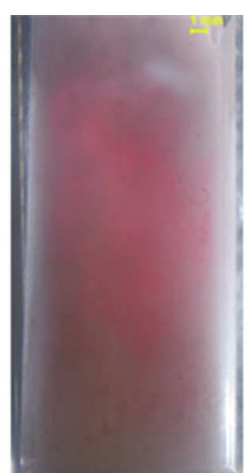

(c) $t=20 \mathrm{~min}$

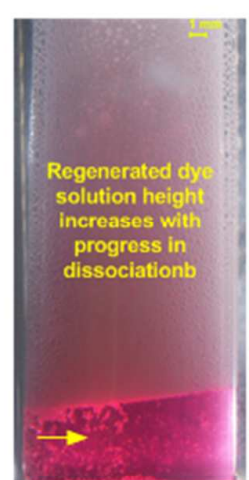

(i) $\mathrm{t}=240 \mathrm{~min}$

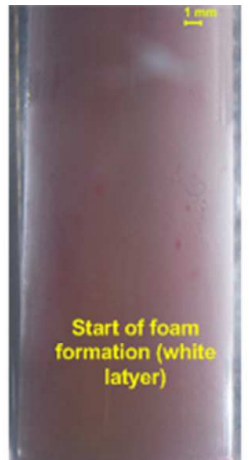

(d) t=35 $\mathrm{min}$

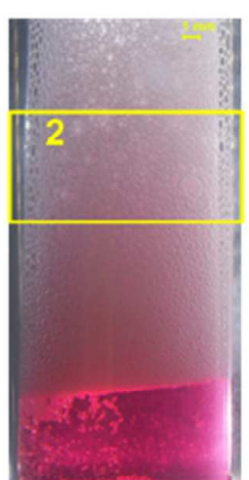

(j) t=420 min

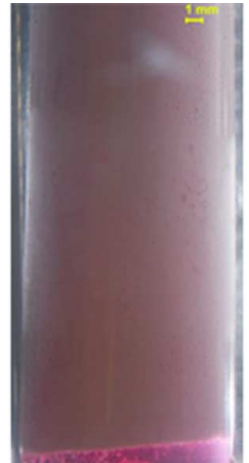

(e) $\mathrm{t}=40 \mathrm{~min}$

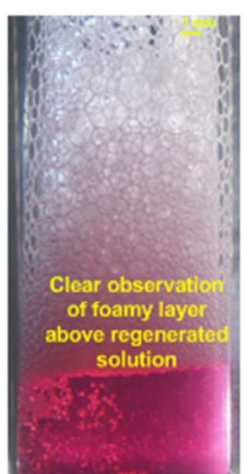

(k) $t=600 \mathrm{~min}$

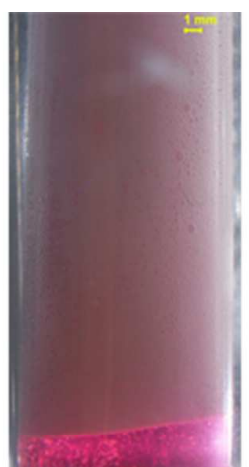

(f) $\mathrm{t}=60 \mathrm{~min}$

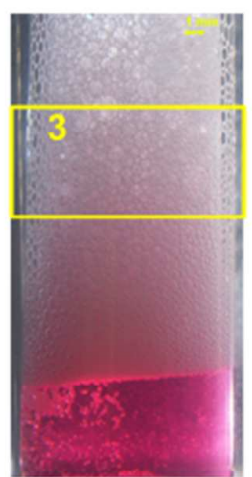

(I) $t=1200 \mathrm{~min}$
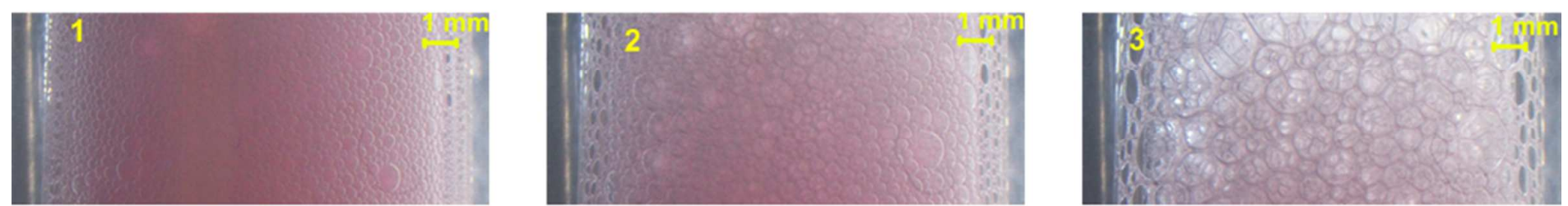

Zoomed insets clearly showing the foam formation; the spherical bubbles in foamy layer (1) grow in size with progress in dissociation time and tend to become hexagonal honey comb like structures

Figure S4. Morphology observations during methane hydrate dissociation using $0.3 \mathrm{wt} \%$ SDS solution in presence of red dye with clear observation of foam formation. 


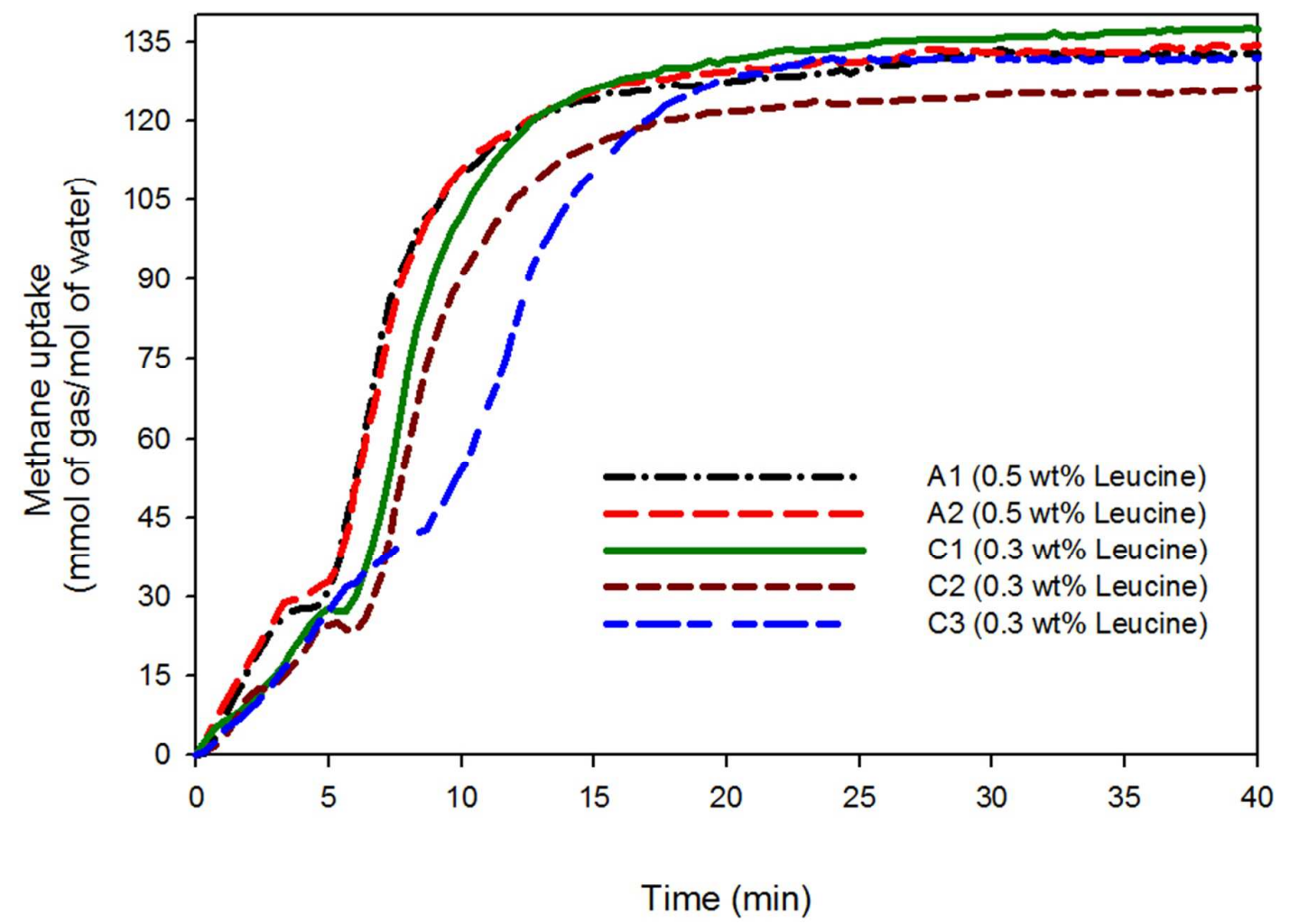

Figure S5. Gas uptake profiles for experiments performed using $0.5 \mathrm{wt} \%$ (trials A1-A2) and $0.3 \mathrm{wt} \%$ leucine (trials C1-C3) solutions 